EPJ Web of Conferences 114, 02073 (2016)

DOI: 10.1051/epjconf/201611402073

(C) Owned by the authors, published by EDP Sciences. 2016

\title{
lonic wind measurements on multi-tip plasma actuators
}

\author{
F. Messanelli ${ }^{1, a}$ and M. Belan ${ }^{1}$ \\ ${ }^{1}$ Politecnico di Milano, Dipartimento di Scienze e Tecnologie Aerospaziali, via La Masa 34, 20156, Milano, Italia
}

\begin{abstract}
This work presents an experimental investigation about the effects of triangular tips on the active electrodes of plasma actuators. The tests are performed on two sets of actuators, corona and DBD, parameterized by means of the tip sharpness and the tips number per unit length. A total number of 30 actuators is considered. The devices are evaluated on the basis of the far field ionic wind velocity, that has been chosen as a representative test common to both kinds of actuator. The dataset includes velocity profiles and maps, that can be integrated to give mass flows and electromechanical efficiencies. Some results are also presented in the parameter space defined by tip sharpness and tips number per unit length: this gives the chance of defining optimal electrode shapes within each set. In general, the longitudinal velocity of the gas increases downstream of the tips in all the actuators tested, but the velocity field is modified to different extents in the two kinds of actuators, and is more complicated for the DBDs than for the coronas. The tips also increase the efficiency of all the actuators, particularly for the corona set, where even the stability is remarkably improved.
\end{abstract}

\section{Introduction}

The present work deals with some properties of the plasma actuators, widely studied because of their capacity to produce a ionic wind. These devices may be operated with direct (corona actuators) or alternate (DBD actuators) current. The induced gas velocity can be used for several flow control applications, as described in the review by Moreau [1], and specifically for the DBD by Corke et al. [2], Wang et al. [3] and Benard et al. [4].

In particular, this work describes an experiment about plasma actuators, both corona and DBD types, in order to characterize the flow field induced by a multi-tip geometry of the upstream electrodes. The tips are triangular, so that the geometry of the electrodes under study can be considered as a generalized form of the serrated edge shape. This shape has already been studied in some previous works for DBDs [5, 6], but a proper parametric study of the relevant geometry has never been performed, while it is completely innovative for corona devices, to the best of our knowledge.

Two sets of actuators, corona and DBD, including 30 different devices, are considered in this experiment. A parametric study is carried out identifying the different actuators by their tip sharpness $r$ (defined as the ratio between tip length and width) and tips number per unit length $n$. Straight edge reference actuators (wire-to-plate for corona, plate-to-plate for DBD) are also tested in order to have a term of comparison. The actuators are evaluated on the basis of the far field ionic wind velocity, measured by means

a e-mail: federico.messanelli@polimi.it of a pitot probe. The devices under test are located on a flat plate in still air and the induced longitudinal velocity is measured across two planes normal to the ionic wind, at distances far enough to avoid the strong tridimensionality of the near field. Current and voltage waveforms for every actuator tested are also acquired, in order to evaluate the power consumption and the electro-mechanical efficiency.

The available data make possible several comparisons among different actuators, within each set and also between actuators of different sets (corona vs DBD). The results exposed below indicate that in general the multi-tip geometry gives better performance with respect to the straight geometry, both for corona and DBD, considering the intensity of the induced ionic wind and the mass flow. Power consumption considerations indicate that corona actuators are more efficient than DBDs. Furthermore, the multi-tip geometry increases corona stability, reducing glow-to-arc transition and favouring the ignition of the discharge.

\section{Experiment setup}

The corona actuators under study are represented in figure 1 and listed by their geometrical parameters in table 1: the set includes a wire-to-plate actuator C1 (figure 1a) as a reference, several actuators with serrated anodes $\mathrm{C} 2 \ldots \mathrm{C} 12$ (figure $1 \mathrm{~b}$ ) and an actuator with isolated tips $\mathrm{C} 13$ (figure 1c). Gap $g$ and cathode size $c$ are common to all the actuators, as reported in figure 1. The specific geometrical parameters are the tip height $h$ and the tip width $w$, listed in table 1 together with the tip sharpness $r=h / w$ and 
(a)

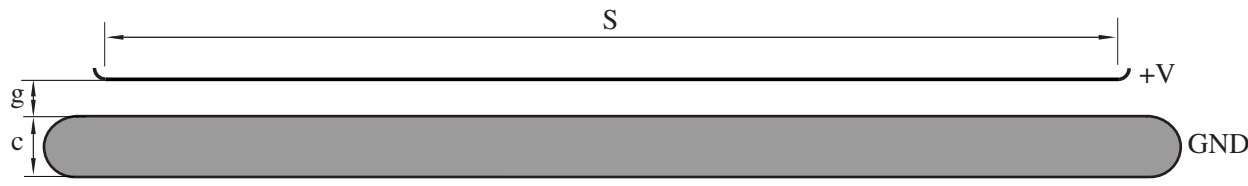

(b)

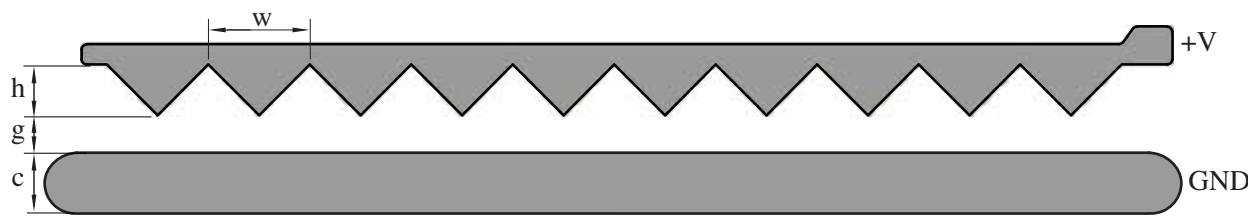

(c)

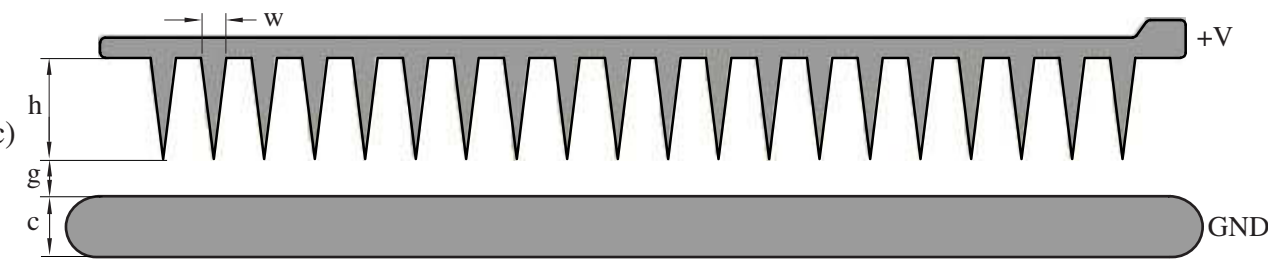

Fig. 1: Geometry of the corona actuators under study: (a) reference wire-plate; (b) multi-tip, serrated; (c) multitip, isolated tips. All the actuators have gap $g=15 \mathrm{~mm}$ and cathode size $c=24 \mathrm{~mm}$. The span is $S=400 \mathrm{~mm}$ for all the actuators except the reference (a), which is $35 \mathrm{~mm}$ wide to ensure its operational stability.

the tips number per unit length $n=N / S=$ (number of tips)/span. The span is $S=400 \mathrm{~mm}$ for all the actuators except the reference wire-plate (figure 1a), that can not be operated in still air over such a span because of its poor stability. This actuator has $S=35 \mathrm{~mm}$ to avoid excessive transitions to spark, so that in figure $1 \mathrm{a}$ is sketched out of scale. The 2 actuators labeled $\mathrm{Cx}$ and $\mathrm{Cxx}$ in table 1 have been prepared for the tests but later excluded because of their poor stability, similar to the wire-plate case $\mathrm{C} 1$. All the corona actuators are operated in the streamer regime, thanks to a suitable high voltage driver. The operating voltage is $V_{0}=17.2 \pm 0.2 \mathrm{kV}$, except for the wire-plate device, that requires $V_{0}=16 \pm 0.1 \mathrm{kV}$ to ignite correctly with the same gap width $g$ as the other actuators. The HV driver-actuator connection is made through a low-pass capacitive filter $(R=4.4$ $\mathrm{M} \Omega, C=0.9 \mathrm{nF}$ ) and a ballast resistor $R_{b}=4.4$ $\mathrm{M} \Omega$. Voltage measurements are obtained by means of a 1:1000 voltage divider (load $47 \mathrm{M} \Omega$ ), current measurements by a shunt resistor $\left(R_{s}=100 \Omega\right)$ on the ground electrode of the actuator under test.

The DBD actuators are represented in figure 2 and listed by their geometrical parameters in table 2 : the set includes a plate-to-plate actuator D1 (figure 2a) as a reference, several actuators with serrated anodes D2...D14 (figure 2b) and an actuator with isolated tips D15 (figure 2c). Span $S$ and ground plate size $c$ are common to all the actuators, as reported in figure 2 . The ground plate is always aligned with the roots of the tips. As for the corona actuators, table 2 lists the specific geometrical parameters, namely tip height and width $h$ and $w$, tip sharpness $r=h / w$ and tips number per unit length $n=N / S$. The DBD actuators are operated by an oscillator-amplifier-output transformer chain, purposely built. All measurements have
Table 1: Parameters of the corona actuators under test.

\begin{tabular}{|r|rr|l|r|}
\hline Name & $h(\mathrm{~mm})$ & $w(\mathrm{~mm})$ & $r=h / w$ & $n=N / S\left(\mathrm{~m}^{-1}\right)$ \\
\hline C1 & 0 & - & 0 & - \\
Cx & 3 & 10 & 0.3 & 100 \\
Cxx & 5 & 10 & 0.5 & 100 \\
C2 & 8 & 4 & 2 & 250 \\
C3 & 8 & 5 & 1.6 & 200 \\
C4 & 10 & 10 & 1 & 100 \\
C5 & 15 & 10 & 1.5 & 100 \\
C6 & 20 & 4 & 5 & 250 \\
C7 & 20 & 5 & 4 & 200 \\
C8 & 20 & 8 & 2.5 & 125 \\
C9 & 20 & 10 & 2 & 100 \\
C10 & 20 & 20 & 1 & 50 \\
C11 & 30 & 10 & 3 & 100 \\
C12 & 40 & 10 & 4 & 100 \\
C13 & 40 & 10 & 4 & 50 \\
\hline
\end{tabular}

been carried out with a sinusoidal waveform having a peak-to-peak voltage $V_{p p}=20 \pm 0.5 \mathrm{kV}$ with frequency $f=815 \pm 5 \mathrm{~Hz}$. The measurement section (voltage divider and current shunt) is the same as for corona actuators.

All the voltage and current waveforms have been acquired at sampling frequencies of 100 Msamples/s. The accuracy of the electrical measurements, reported for the power consumption of the devices, is in the order of $0.4 \mathrm{~W}$ (or $1 \mathrm{~W} / \mathrm{m}$ ) for the coronas and $1.2 \mathrm{~W}$ (or $3 \mathrm{~W} / \mathrm{m}$ ) for the DBDs.

Sizes and operating conditions for the two sets have been selected after preliminary tests in order to give ionic wind velocities in the same order of magnitude, thus comparisons within each set are possible as well as comparisons between actuators in different 
(a)

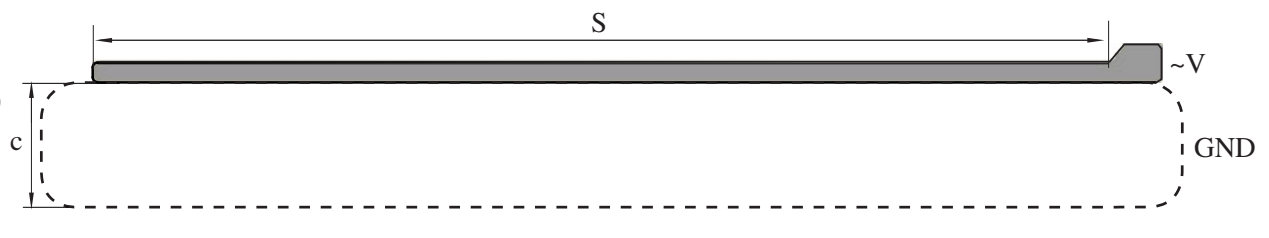

(b)

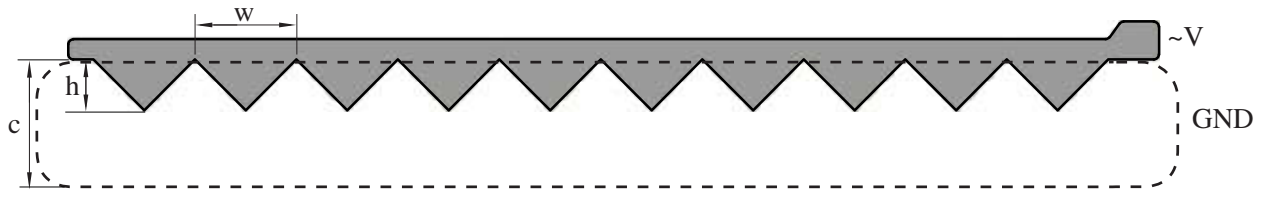

(c)

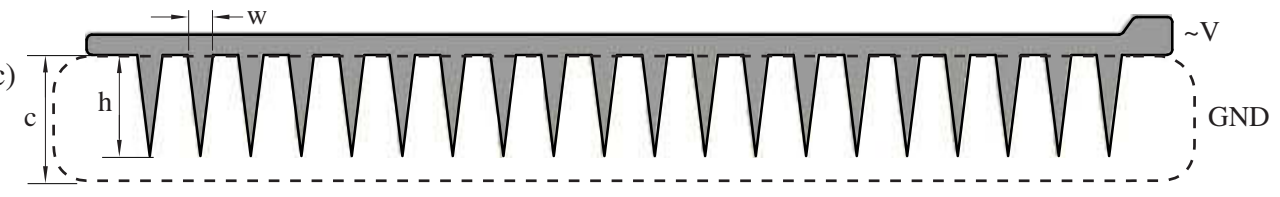

Fig. 2: Geometry of the DBD actuators under study: (a) reference plate-plate; (b) multi-tip, serrated; (c) multitip, isolated tips. All the actuators have span $S=400 \mathrm{~mm}$ and ground plate size $c=50 \mathrm{~mm}$.

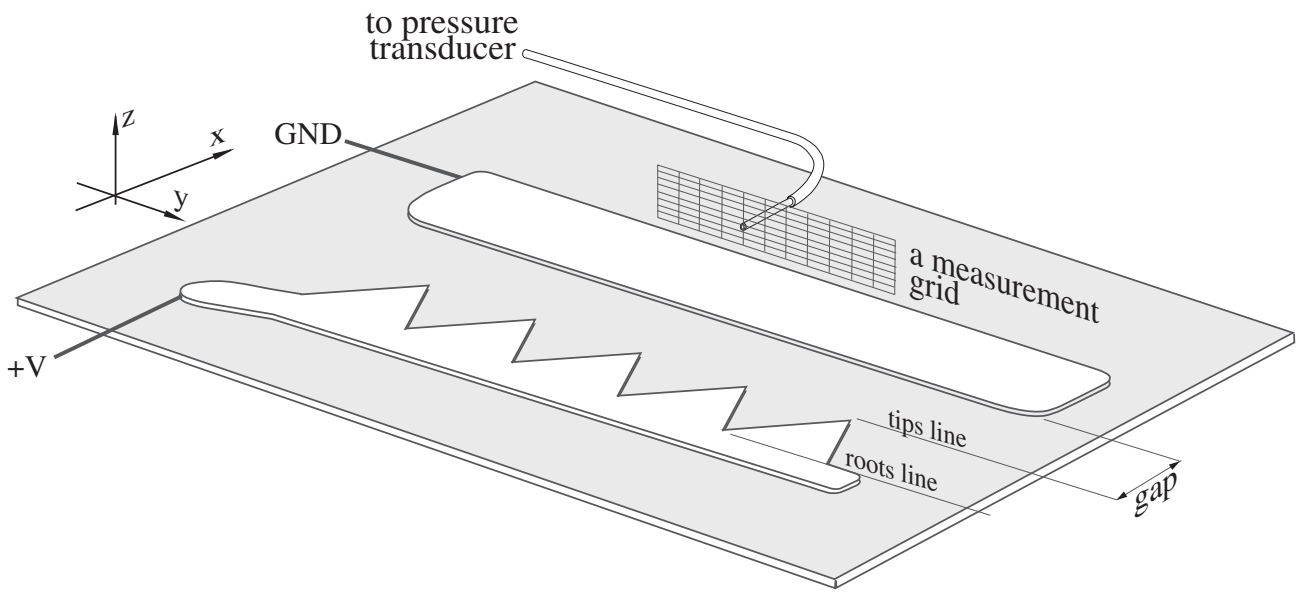

Fig. 3: Experimental setup, shown for a multi-tip corona actuator (non-scaled for clarity). The setup for DBDs is the same, but the grounded electrode is under the PMMA flat plate that serves as dielectric and is aligned with the roots.

sets. The actuators, placed on a flat plate in still air, are evaluated on the basis of the far field ionic wind velocity, measured by means of an insulating pitot probe as in figure 3, which shows also the reference system $x y z$. The pressure probe has an outer diameter $d_{o}=0.8 \mathrm{~mm}$, an inner one $d_{i}=0.4 \mathrm{~mm}$ and is connected to a differential pressure transducer with range $\pm 200 \mathrm{~Pa}$ and accuracy $0.05 \mathrm{~Pa}$. The induced longitudinal velocity is measured across different normal planes $y z$ at distances $x=17 \mathrm{~mm}$ and $40 \mathrm{~mm}$ from the tips line of the active electrode; this ensures that the field of motion is far enough from the tips as to have a ionic wind direction close to the $x$-direction, and in particular within the directional error band of the probe, that is less than $1 \%$ for misalignments within $34^{\circ}$ around the probe axis. The velocities are then ob- tained through the equation

$$
p_{m}-p=\frac{1}{2} C_{p} \rho v^{2},
$$

where $p_{m}$ is the measured pressure, $p$ the reference atmospheric pressure and $C_{p}$ a coefficient including the experimental corrections, purposely set to maintain the $1 \%$ pressure accuracy even when the Reynolds number is very low and/or the probe touches the wall [7]. The humidity during the tests has been maintained to $22 \pm 5 \%$. The gas density has been determined by the atmospheric pressure and temperature through the state law, assuming a negligible plasma heating. The measurements system described above gives uncertainties on the velocity data in the order of $0.1 \mathrm{~m} / \mathrm{s}$, excluding error sources due to small irregularities of the electrodes shapes. Furthermore, the unlikely case of large misalignments in the far field can be treated by means of the calibration curves of 
Table 2: Parameters of the DBD actuators under test.

\begin{tabular}{|r|rr|l|r|}
\hline Name & $h(\mathrm{~mm})$ & $w(\mathrm{~mm})$ & $r=h / w$ & $n=N / S\left(\mathrm{~m}^{-1}\right)$ \\
\hline D1 & 0 & - & 0 & - \\
D2 & 3 & 10 & 0.3 & 100 \\
D3 & 5 & 5 & 1 & 200 \\
D4 & 5 & 10 & 0.5 & 100 \\
D5 & 10 & 10 & 1 & 100 \\
D6 & 15 & 10 & 1.5 & 100 \\
D7 & 20 & 5 & 4 & 200 \\
D8 & 20 & 10 & 2 & 100 \\
D9 & 20 & 20 & 1 & 50 \\
D10 & 20 & 25 & 0.8 & 40 \\
D11 & 20 & 40 & 0.5 & 25 \\
D12 & 25 & 10 & 2.5 & 100 \\
D13 & 30 & 10 & 3 & 100 \\
D14 & 35 & 10 & 3.5 & 100 \\
D15 & 40 & 10 & 4 & 50 \\
\hline
\end{tabular}

the probe: for angular differences between the velocity vector and the probe axis up to $\pm 40^{\circ}$, the uncertainty for the longitudinal velocity component cannot exceed values of $0.2 \mathrm{~m} / \mathrm{s}$.

\section{Results}

\subsection{Corona stability}

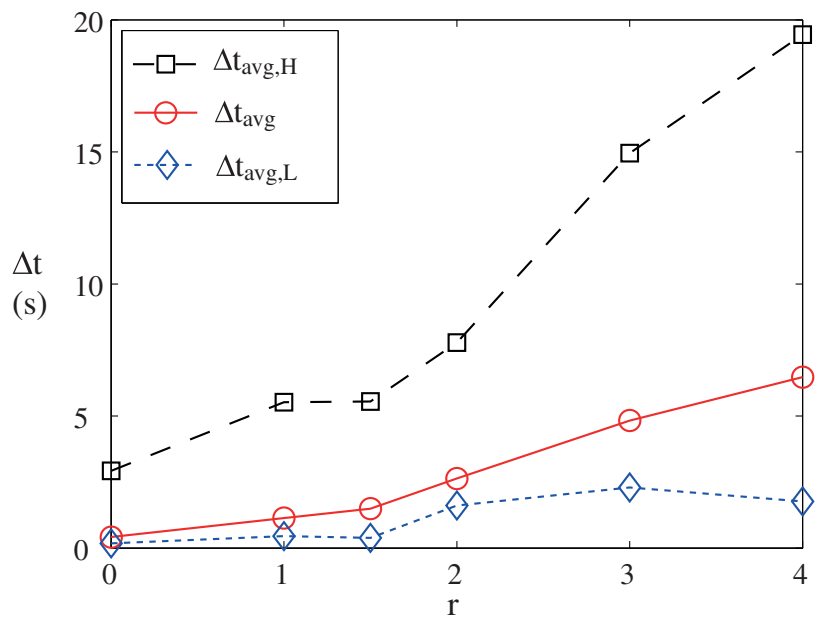

Fig. 4: Mean times between transient sparks for corona actuators $(\mathrm{C} 1,4,5,9,11,12)$ of increasing sharpness $(r=0$ to 4$)$.

In this work most data about corona actuators have been acquired thanks to their improved stability, that can be related to the geometry under study, as shown in what follows. In general, the sparks are visible to the naked eye but they can also be recorded as short and strong current pulses, in the order of $1 \mathrm{~A}$ and more. The power supply can not afford a permanent transition to the arc regime, so that this phenomenon can be described as a 'transient spark' [8], sustained only by the occasional complete discharge of the circuit capacity. The stability of a corona under steady working conditions can be quantified by reporting the time $\Delta t$ between consecutive transient sparks as in figure 4: this figure is obtained from the histograms of the $\Delta t$ measured along tests including hundredth of events for a set of actuators of sharpness growing from $r=0$ to 4, namely $\mathrm{C} 1,4,5,9,11$ and 12. The histogram for a given actuator has in general a peak corresponding to relatively short $\Delta t$ and a wide tail representing long $\Delta t$. The relevant information is shown in figure 4 by the lower and upper curves. The former is the mean time $\Delta t_{a v g, L}$ of the lower part of the histograms, below the median value; the latter is the mean time $\Delta t_{a v g, H}$ of the upper part of the histograms. The figure is completed by the central curve, which is the standard mean time $\Delta t_{\text {avg }}$, and shows a clear increase of the time between sparks as the tip sharpness grows. This property should be related to the anode shape, since the sparks can start only from the small tip regions, and to the relevant local field, that grows with $r$ and makes the discharge less sensitive to perturbations. As a final remark, it is important noting that even if the trend of the data is significant, the $\Delta t$ values are specifically related to the present setup through its physical parameters as the circuit capacity.

\subsection{Flow velocity data}

This section presents some examples of velocity data, showing the ionic wind dependence on the geometry of the tips. The flow is periodical in the span direction, with slight differences between different tips, due to very small imperfections in the realization process. However, the values presented here are obtained as an average on at least 2 different tips. The velocity curves along the $z$-axis for three different span locations, corresponding to a tip, a root and halfway between them, and two different distances along the $x$-axis (17 and $40 \mathrm{~mm}$ from the tips line, see figure 3 ) are presented in figures $5 \mathrm{a}, \mathrm{b}$ and $6 \mathrm{a}$,b for two representative actuators, corona C11 of table 1 and DBD D13 of table 2, that share the same geometry: $r=3, n=100$. Furthermore, the velocity maps over the cross-section $y z$ for the same distances along the $x$-axis are presented in figures $5 \mathrm{c}, \mathrm{d}$ and $6 \mathrm{c}, \mathrm{d}$ for two tips. The measurements locations are marked with black dots. Obviously, the flow pattern is periodical over all the other tips, with minor exceptions for the extreme tips, due to border effects.

For the corona actuator in figure 5 the maximum of the velocity is measured downstream of the tips at a height $z=3.75 \mathrm{~mm}$ for $x=17 \mathrm{~mm}$ and $z=3.9 \mathrm{~mm}$ for $x=40 \mathrm{~mm}$. The $2 \mathrm{D}$ velocity maps show the momentum diffusion as $x$ grows, whereas the position of the velocity maximum remains quite far from the wall. This behaviour is similar for all the multi-tip corona tested, instead the wire-to-plate reference actuator has its maximum located nearer to the wall, at $z=2.4 \mathrm{~mm}$ for $x=17 \mathrm{~mm}$. The velocities measured downstream 
of the tips are larger than downstream of the roots, because the corona discharge originates only from the anode tips and expands like a fan towards the cathode. However, due to the diffusive mechanisms, the differences between tips and roots decrease when moving along the $x$-axis, as it can be seen comparing figure $5 \mathrm{a}, \mathrm{c}$ and $\mathrm{b}, \mathrm{d}$. The velocities induced by the multi-tip actuators are larger than for the straight wire-to-plate: the tips are regions of high electric field and may cause an increase in the ionization efficiency. Indeed, the region close to the tip is characterized by a very strong luminosity, much more than close to the wire anode in the wire-to-plate actuator, implying that a high concentration of ions is achieved. This phenomenon is observed also for the DBDs: even if in that case the discharge originates along the whole electrode perimeter, the luminosity, and consequently the ion concentration, is higher in the proximity of the tips.

Confronting the DBD curves and maps in figure 6 with the corona ones of Fig. 5, the induced flow appears more complex for the DBDs. At the nearest $x$ distance $(17 \mathrm{~mm})$, once again the velocity maximum is found downstream of the tips, but at a lower height, $z=0.65 \mathrm{~mm}$. This value is the same for all the DBD tested, with the exception of the straight edge, that gives $z=0.9 \mathrm{~mm}$. This great difference between coronas and DBDs may be due to the different morphology of the electric field between the electrodes: the field lines are more flattened toward the wall for the DBDs, while they are more curved and extended in the $z$-direction for the coronas. The velocities are lower for the DBD, so that, as it will shown later, the DBDs require a larger power to produce a given ionic wind, but the location of the velocity maximum nearer to the wall may be better for the boundary layer control. Another property of the multi-tip DBDs of importance in flow control, known in literature (e.g.[5]), is the induction of a strongly three-dimensional flow since the discharge is generated along the whole active electrode perimeter and the induced velocity is locally normal to the electrode edge. This implies that in each space between adjacent tips two oppositely directed jets are generated, that collide in the middle of the region, downstream of the root; here a vertical component, directed away from the wall, is created, while in the regions behind and over the tips the flow moves toward the wall, for continuity reasons. Thus, a DBD creates a ionic wind made of a longitudinal component interacting with transverse components that produce periodic pinch-spread effects on the streamlines and a distribution of streamwise vorticity. This can be seen in the curves and maps of figure 6 , where the velocity downstream of the roots has a consistent extension in the $z$-direction, as well as a maximum located at a larger distance from the wall. Furthermore, for increasing distances along the $x$-axis, the velocity maximum downstream of the roots may grow up to reach the same order of the one downstream of the tips (figure $6 \mathrm{~b}$ and $\mathrm{d}$ ): this phenomenon, already described in literature [9], may be due to the streamlines spreading over the tip zones and pinching over the root (a)

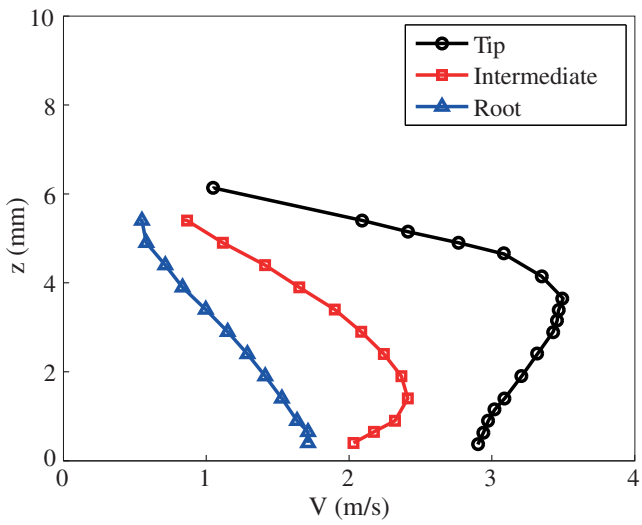

(b)
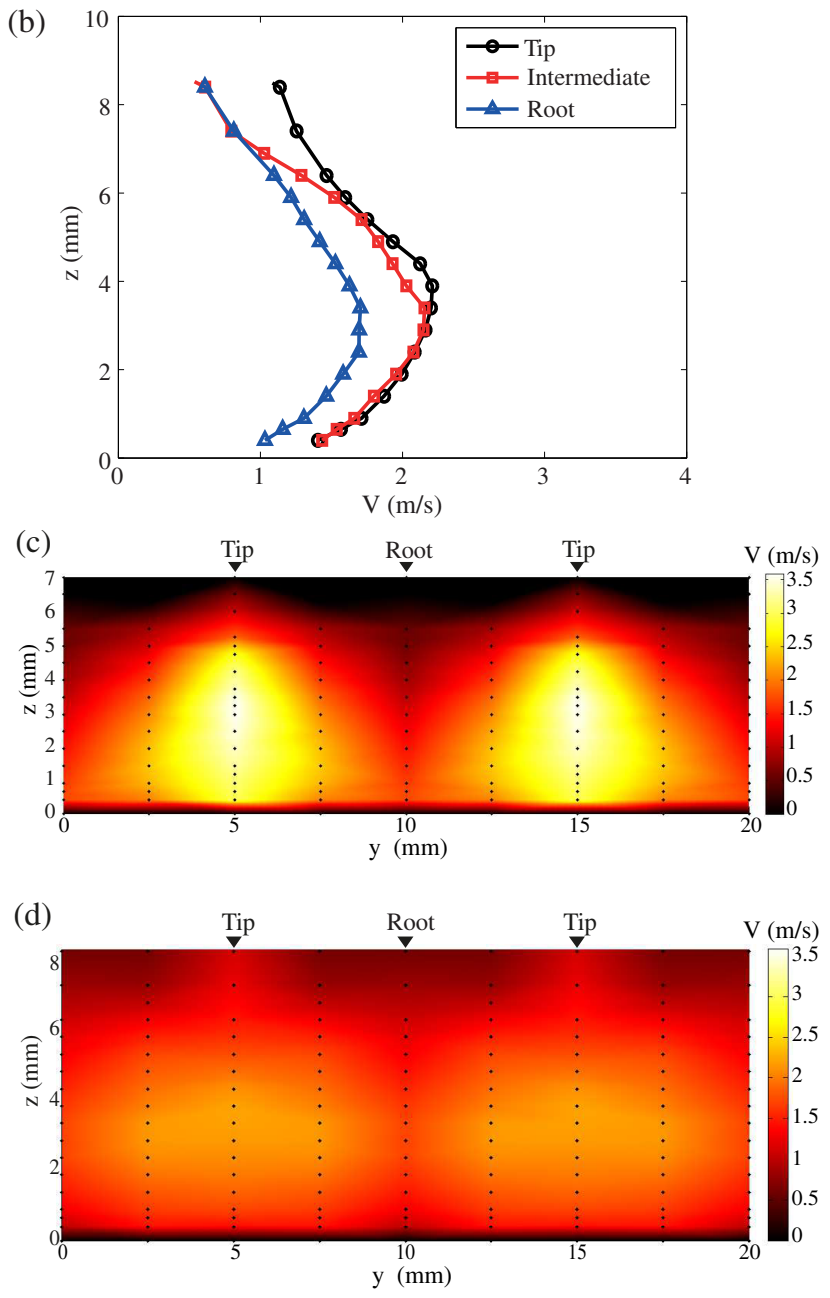

Fig. 5: Corona actuator C11: velocity curves at $x=17$ $\mathrm{mm}$ (a) and $40 \mathrm{~mm}$ (b); velocity maps at $x=17 \mathrm{~mm}$ (c) and $40 \mathrm{~mm}(\mathrm{~d})$. Maps interpolated and completed by no-slip condition.

zones, where the higher velocities streamlines tend to coalesce while the flow develops along the $x$ direction.

\subsection{Data in the parameter space}

The properties of the actuators under study can be considered as dependent on the actuator location over 
(a)

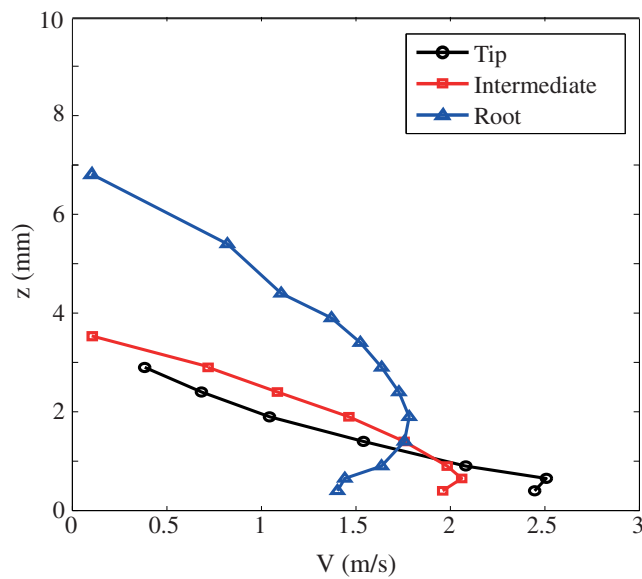

(b)
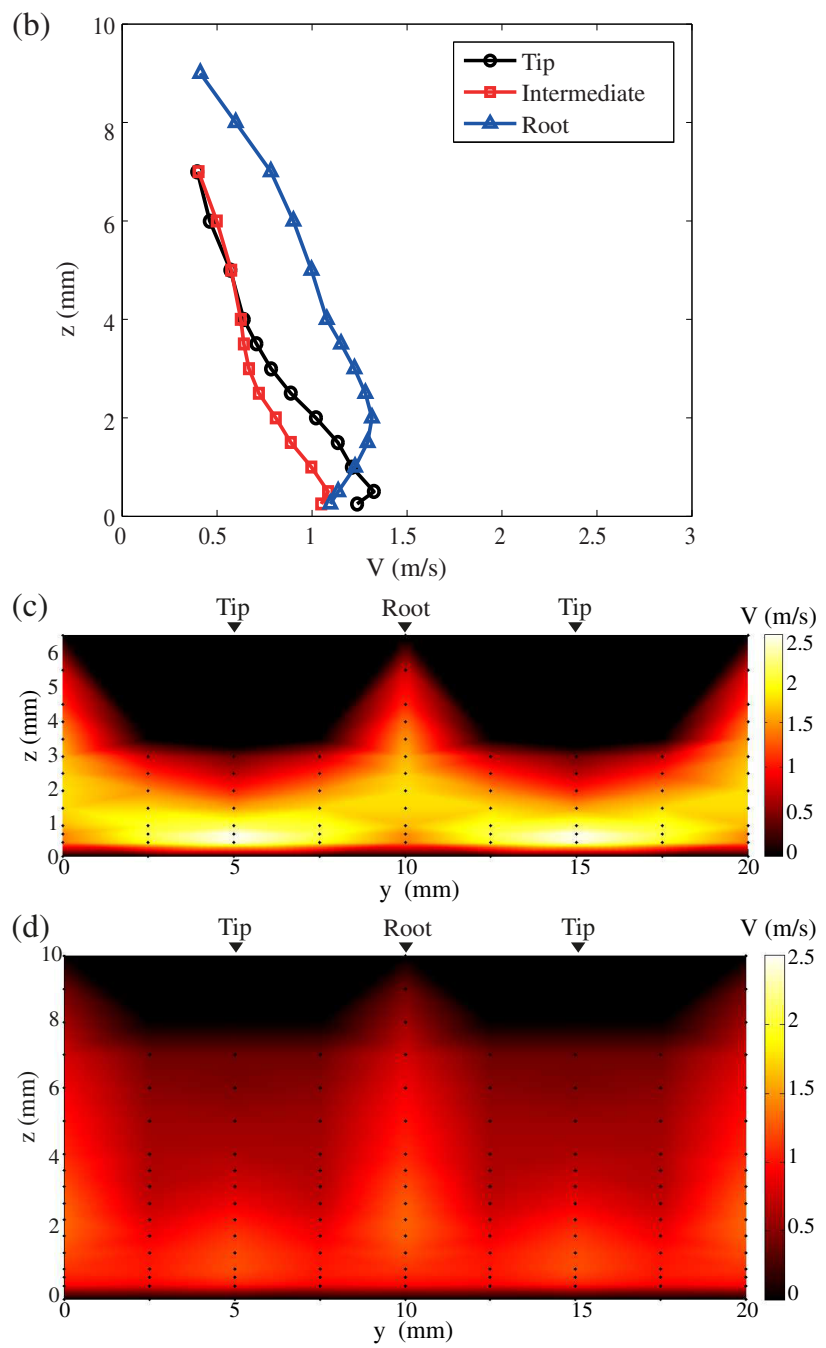

Fig. 6: DBD actuator D13: velocity curves at $x=17$ $\mathrm{mm}$ (a) and $40 \mathrm{~mm}$ (b); velocity maps at $x=17 \mathrm{~mm}$ (c) and $40 \mathrm{~mm}$ (d). Maps interpolated and completed by no-slip condition.

the parameter space $(n, r)$, i.e. as function of the parameters $r=h / w$ (tip sharpness) and $n=N / S$ (tips number per unit length) introduced in $\S 2$. Figures 7 and 8 present a comparison of 3 coronas (C6,C9, C10) and 3 DBDs (D7, D8, D11) lying in the param- eter space $(n, r)$ along the same oblique straight line $r=0.02 n$. This line represents actuators having the same tip length $h=20 \mathrm{~mm}$ but different widths $w$. It can be seen that the velocity difference downstream of tips and roots increases when $w$ grows, i.e. when the tips are more spaced. Also the values of the maximum velocity increase. It seems that more isolated tips are more efficient in generating high velocities, whereas, if the tips are too close each other, their beneficial effects are partially lost and the behaviour may become similar to that of the straight actuator. However, it is important noting that the characterization of an actuator by means of the maximum velocity induced locally can not be considered as complete; in what follows, all the actuators will be compared on the basis of a reliable global parameter.

Figure 9 shows the global mass flows produced by all the actuators under test, obtained by integration of the velocity profiles over the measurement grids, at $x=17 \mathrm{~mm}$. The mass flow is an important parameter, even more important than the maximum velocity, to characterize the effectiveness of a given actuator for flow control purposes. For corona actuators (figure $9 \mathrm{a})$, the performance improves increasing the sharpness $r$ : the more acute the tips are, the more intense is the electric field in their proximity and thus their performance. The optimal mass flow is achieved for the actuator $\mathrm{C} 12(n=100, r=4)$, that has very sharp tips but not so numerous. It is probable that excessively increasing the parameter $n$ leads to very close ionization regions, and the electric field eventually becomes similar to the one of the wire-to-plate, thus losing the beneficial effects of the tips. On the other hand, the point at $n=50, r=4$ shows that tips too distant each other worsen the performance, so the best mass flow arises as a balance between the sharpness and the numerosity of the tips. For the DBDs (figure $9 \mathrm{~b}$ ), the highest mass flow is obtained for the D7 $(n=200, r=4)$ : also in this case an increase of the sharpness leads to better performance, but the proximity of tips is not so negative as for the corona actuators, even if it reduces the maximum induced velocity. This is confirmed by the good performance achieved by actuator D3 $(n=200, r=1)$, that is also located on the right border of the figure. This may be due to the fact that the active perimeter for DBDs is very extended for a high number of tips, so that the ionic wind generated between tips compensates the lower velocity maxima with respect to the case of more isolated tips. In conclusion, coronas induce mass flows larger than DBDs, which is good for flow control purposes, but the velocity maxima are more distant from the wall, that is a negative characteristic for boundary layers control.

Electrical measurements have also been performed, in order to quantify the electrical power consumed by the actuators and the electro-mechanical efficiency, that is defined as the ratio between the available mechanical power and the consumed electrical power. In general, it is found that DBD actuators require a higher power than coronas to produce similar velocities. This reflects also on the electro-mechanical effi- 

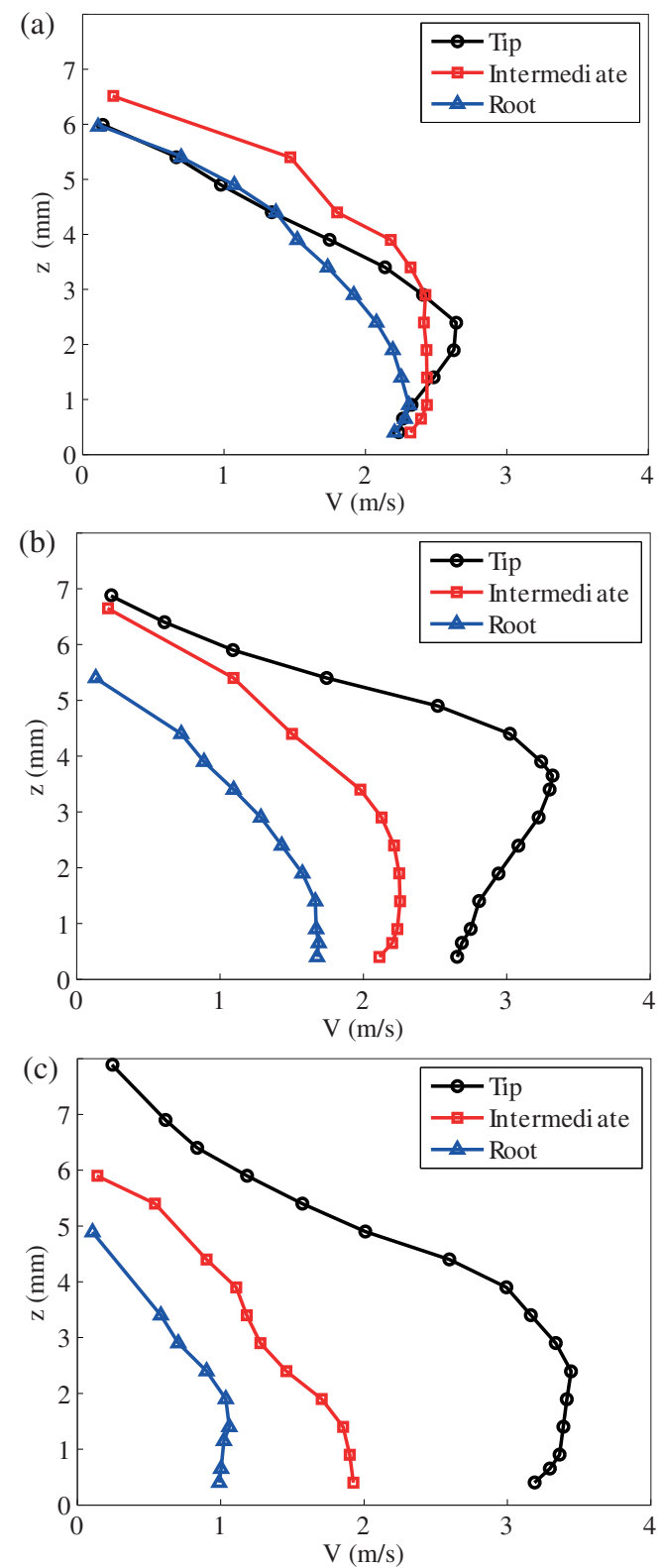

Fig. 7: Velocity curves at $x=17 \mathrm{~mm}$ for the corona actuators C6 (a), C9 (b), C10 (c) having the same $h$ $=20 \mathrm{~mm}$ and growing $w=4,10,20 \mathrm{~mm}$.

ciency: the corona actuator $\mathrm{C} 11$, whose velocity curves are shown in figure 5 , has an efficiency of $0.96 \%$, while the DBD D13 of figure 6 has an efficiency of $0.05 \%$, that is remarkably lower. Nevertheless, it must be noticed that the efficiencies of the straight reference actuators, that are about $0.05 \%$ for the corona wireto-plate and $0.04 \%$ for the DBD plate-to-plate, are lower than the ones of almost every multi-tip actuator tested. In particular, the efficiency improvement for corona actuators is about two order of magnitude, while it is less substantial, but still present, for the DBDs.
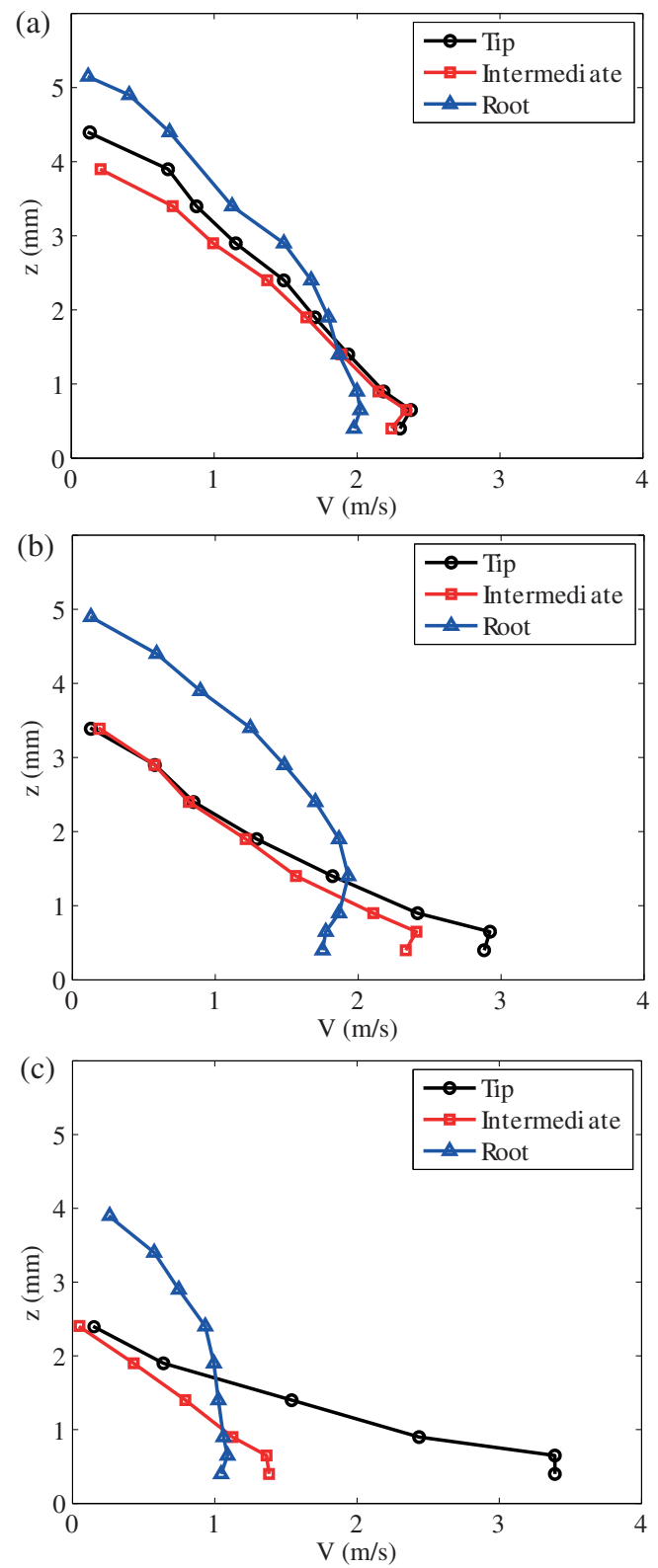

Fig. 8: Velocity curves at $x=17 \mathrm{~mm}$ for the DBD actuators D7 (a), D8 (b), D11 (c) having the same $h$ $=20 \mathrm{~mm}$ and growing $w=4,10,20 \mathrm{~mm}$.

\section{Conclusions}

In this work, velocity measurements downstream of two sets of multi-tip plasma actuators, both corona and DBD types, have been performed. Reference straight actuators have also been tested. The geometry of the upstream electrodes was characterized by the tip sharpness $r$ and the tips number per unit length $n$. For the corona set, $r$ has been varied between 0 and 5 , while $n$ between 50 and 250; for the DBD set, $r$ was investigated between 0 and 4 , and $n$ between 25 and 200 . The measured profiles show that the induced velocity is not uniform in the span direction: for coronas, it is periodic and higher downstream of the tips, thanks to the increased ionization intensity in the near-tip regions; for DBDs, the velocity field is also periodic 

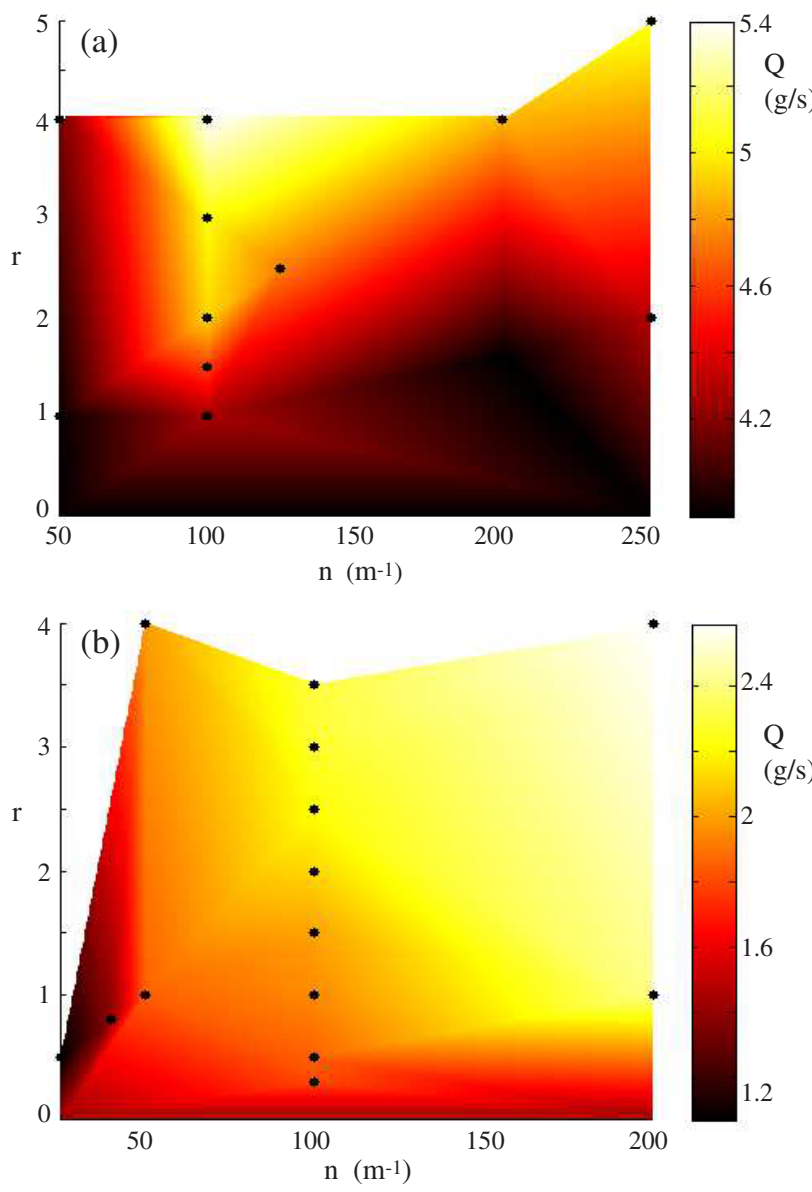

Fig. 9: Mass flow rate $Q$ vs. tip number per unit length $n$ and tip sharpness $r$ for the tested corona (a) and DBD (b) actuators (interpolated data). Each actuator is represented by a dot on the map, except the straight ones, which corresponds to the horizontal axes.

but more complicated, since in the near field of these actuators the induced velocity vector is locally normal to the perimeter of the exposed electrode. A remarkable effect is the generation of a couple of streamwise counter-rotating vortices downstream of each space between adjacent tips, with possible beneficial effects for flow control purposes, as reported in [5].

Optimal geometries within those tested have also been determined in terms of induced mass flow. For the corona actuators, the maximum mass flow has been registered for very sharp, but not so numerous tips (actuator C12). The tips are regions of high electric field, and thus of high ionization rate. Generally speaking, the performance improves by increasing the tip sharpness. However, an excessive number of tips (i.e. tips very close each other) may lose these benefits, leading to a condition more similar to the straight wire-to-plate, that has the worst performance among the actuators tested. On the other hand, since the corona discharge is generated only from the anode tips, an insufficient number of tips may also be detrimental for performance. For the DBD set, the maximum mass flow has been measured for very sharp and numerous tip (actuator D7): in this case a great num- ber of tips implies a very extended active perimeter, since the discharge is generated on the whole active edge of the exposed electrode.

In general, it is important to notice that almost all the multi-tip actuators under test have achieved better performance in terms of induced velocity and mass flow with respect to the straight reference actuators. In particular, thanks to the different characteristics of the electric fields, coronas induce the maximum velocity at a higher distance from the wall than DBDs. This may not be a positive characteristics for boundary layer control. However, multi-tip corona actuators require less power than DBDs to induce larger mass flows: the electro-mechanical efficiency is higher. This feature, in addition to the improved discharge stability with the multi-tip geometry, is very promising.

\section{References}

1. E. Moreau, J. Phys. D: Applied Physics 40, 605636 (2007)

2. T.C. Corke, C.L. Enloe, S.P. Wilkinson, Annual Rev. Fluid Mech. 42, 505-529 (2010)

3. J.J. Wang, K.S. Choi, L.H. Feng, T.N. Jukes, R.D. Whalley, Prog. Aerosp. Sci. 62, 52-78 (2013)

4. N. Benard, E. Moreau, Exp. Fluids 55: 1846 (2014)

5. R. Joussot, A. Leroy, R. Weber, H. Rabat, S. Loyer, D. Hong, J. Phys. D: Appl. Phys. 46: 125204 (2013)

6. F. O. Thomas, T.C. Corke, M. Iqbal, A. Kozlov, D.Schatzman, AIAA J. 47, 2169-2178 (2009)

7. C. Tropea, A.L. Yarin, J.F.Foss (Eds.), Springer Handbook of Experimental Fluid Mechanics, Section 5.1.1 (Springer-Verlag, Heidelberg, 2007)

8. M. Janda, V. Martišovitš, Z. Machala, Plasma Sources Sci. Technol. 20: 035015 (2011)

9. S. Roy, C.C. Wang, J. Phys. D: Appl. Phys. 42: 032004 (2009) 